\title{
On normal and non-normal holomorphic functions on complex Banach manifolds
}

\author{
Peter V. Dovbush
}

\begin{abstract}
Let $X$ be a complex Banach manifold. A holomorphic function $f: X \rightarrow \mathbb{C}$ is called a normal function if the family $\mathcal{F}_{f}=\{f \circ \varphi: \varphi \in \mathcal{O}(\Delta, X)\}$ forms a normal family in the sense of Montel (here $\mathcal{O}(\Delta, X)$ denotes the set of all holomorphic maps from the complex unit disc into $X$ ). Characterizations of normal functions are presented. A sufficient condition for the sum of a normal function and non-normal function to be non-normal is given. Criteria for a holomorphic function to be non-normal are obtained.

These results are used to draw one interesting conclusion on the boundary behavior of normal holomorphic functions in a convex bounded domain $D$ in a complex Banach space $V$. Let $\left\{x_{n}\right\}$ be a sequence of points in $D$ which tends to a boundary point $\xi \in \partial D$ such that $\lim _{n \rightarrow \infty} f\left(x_{n}\right)=L$ for some $L \in \overline{\mathbb{C}}$. Sufficient conditions on a sequence $\left\{x_{n}\right\}$ of points in $D$ and a normal holomorphic function $f$ are given for $f$ to have the admissible limit value $L$, thus extending the result obtained by Bagemihl and Seidel.
\end{abstract}

Mathematics Subject Classification (2000): 32A18 (primary).

\section{Introduction}

The idea of associating to a meromorphic function $f$ on the complex unit disc $\Delta=\{z \in \mathbb{C}:|z|<1\}$ a family $\mathcal{F}=\{f \circ g: g \in \operatorname{Aut}(\Delta)\}$, where $\operatorname{Aut}(\Delta)$ is the group of biholomorphic automorphisms of $\Delta$, and of ascribing to the function $f$ properties of the family $\mathcal{F}$ apparently arose in work of Yosida [16] in 1934 and was considered by Noshiro [14] in 1937. In 1957, Lehto and Virtanen [12] defined "normal functions" to be those meromorphic functions $f$ whose associated families $\mathcal{F}$ were normal in the sense of Montel.

Since that time, the subject of normal functions has been studied extensively, resulting in substantial development in the single complex variable context and in generalizations to the setting of several complex variables (see lists of references in $[11,13]$, and [17]).

Received January 30, 2008; accepted in revised form June 29, 2008. 
In this paper, we investigate normal holomorphic functions on complex Banach manifolds. After preliminaries in Section 2, in Section 3 we obtain certain relations existing between notions of normal functions, and $P$-sequences. In Section 4 we give criteria for a holomorphic function to be a non-normal function. In Section 5 we give sufficient condition for the sum of a normal function and not a normal function to be a non-normal function. Finally in Section 6 we investigate the boundary behavior in a fixed boundary point of a normal function defined on a convex bounded domain in a complex Banach space.

\section{Preliminaries}

We refer the reader to the paper [4] and the books [3] and [5] for background on complex analysis in infinite dimension. The notation we use is essentially the same as in [4].

Let $X$ be a complex Banach manifold modelled on a complex Banach space of positive, possibly infinite, dimension; $X$ is assumed to be a connected Hausdorff space. For each $x$ in $X$ the tangent space to $X$ at point $x$ will be denoted by $T_{X}(X)$. The tangent bundle $T(X)$ of $X$ consists of the ordered pairs $(y, v)$ such that $x \in X$ and $v \in T_{X}(X)$. We shall denote the space of all holomorphic maps from the unit disk $\Delta$ into $X$ by $\mathcal{O}(\Delta, X)$.

The infinitesimal Kobayashi pseudometric on the complex Banach manifold $X$ is the function $k_{X}$ on $T(X)$ defined by the formula

$$
k_{X}(x, v)=\inf \left\{|a|: \exists \varphi \in \mathcal{O}(\Delta, X), \varphi(0)=x, \varphi_{*}(0) a=v\right\}
$$

where $\varphi_{*}(0)$ is the linear map induced by $\varphi$ from $T_{0}(\Delta)$ to $T_{\varphi(0)}(X)$.

We say that $k_{X}$ is a metric if $k_{X}(x, v)>0$ for all $(x, v) \in T_{X}(X), v \neq 0$.

The Kobayashi length of a piecewise $C^{1}$ curve $\gamma:[0,1] \rightarrow X$ in $X$ is defined to be the upper Riemann integral

$$
L_{k}(\gamma)=\bar{\int}_{0}^{1} k_{X}\left(\gamma(t), \gamma^{\prime}(t)\right) d t
$$

and the pseudometric $\widetilde{K}_{X}(x, y)$ is the infimum of the lengths of all piecewise $C^{1}$ curves joining $x$ to $y$ in $X$.

For $z_{1}, w_{1}$ in $\Delta$ the Poincaré distance is expressed by

$$
d_{\Delta}\left(z_{1}, w_{1}\right)=\frac{1}{2} \log \frac{1+\left|\frac{z_{1}-w_{1}}{1-\bar{z}_{1} w_{1}}\right|}{1-\left|\frac{z_{1}-w_{1}}{1-\bar{z}_{1} w_{1}}\right|}=\tanh ^{-1}\left(\left|\frac{z_{1}-w_{1}}{1-\bar{z}_{1} w_{1}}\right|\right) .
$$

The Kobayashi pseudometric between two points $x, y$ in $X$ is defined as follows. Consider all finite sequences of points $p_{0}=x, p_{1}, \ldots, p_{k-1}, p_{k}=y$ of $X$ such that 
there exist points $z_{1}, \ldots, z_{k}, w_{1}, \ldots, w_{k}$ of $\Delta$ and maps $\varphi_{1}, \ldots, \varphi_{k} \in \mathcal{O}(\Delta, X)$ satisfying $\varphi_{j}\left(z_{j}\right)=p_{j-1}$ and $\varphi_{j}\left(w_{j}\right)=p_{j}, j=1, \ldots, k$.

The Kobayashi pseudometric $K_{X}(x, y)$ is, by definition,

$$
K_{X}(x, y)=\inf \sum_{j=1}^{k} d_{\Delta}\left(z_{j}, w_{j}\right)
$$

where the infimum is taken over all possible choices of points and maps.

The spherical arc length element $d s$ on the Riemann sphere $\overline{\mathbb{C}}$ is given by

$$
d s(z, d z)=\frac{|d z|}{1+|z|^{2}}
$$

The spherical length

$$
s(\gamma)=\int_{\gamma} d s(z, d z)
$$

of a curve $\gamma$ in $\overline{\mathbb{C}}$ induces a metric in the following manner.

Given distinct points $a, b$ on the Riemann sphere, define

$$
s(a, b)=\inf \{s(\gamma)\}
$$

where the infimum is taken over all piecewise $C^{1}$ curves on $\overline{\mathbb{C}}$ which join $a$ with $b$. Then $s(a, b)$ defines a metric on the sphere known as the spherical metric.

Denote by $\mathcal{O}(X)$ the set of all holomorphic functions on $X$. A family $F \subseteq$ $\mathcal{O}(\Delta)$ is said to be normal in $\Delta$ if every sequence $\left\{f_{n}\right\} \subset F$ has a subsequence which converges uniformly (with respect to the Euclidean metric) on compacta in $\Delta$ or diverges uniformly to $\infty$ on compacta in $\Delta$.

Definition 2.1. A function $f$ in $\mathcal{O}(X)$ is called a normal function if the family $\mathcal{F}=\{f \circ \varphi: \varphi \in \mathcal{O}(\Delta, X)\}$ is normal.

A family $F \subseteq \mathcal{O}(\Delta)$ is said to be spherically equicontinuous at a point $z_{0} \in \Delta$ if for each positive number $\epsilon$ there is a positive number $\delta$ such that $s\left(f(z), f\left(z_{0}\right)\right)<$ $\epsilon$ for $K_{\Delta}\left(z, z_{0}\right)<\delta$ and $f$ in $F$.

Following Gauthier [7] we shall define a sequence $\left\{x_{n}\right\}$ of points in $X$ to be a $P$-sequence of $f \in \mathcal{O}(X)$ if there is a sequence $\left\{y_{n}\right\}$ of points in $X$ such that $K_{X}\left(x_{n}, y_{n}\right) \rightarrow 0$ as $n \rightarrow \infty$ but $s\left(f\left(x_{n}\right), f\left(y_{n}\right)\right) \geq \epsilon$ for some $\epsilon>0$ for each positive integer $n$.

The notion of $P$-sequence was originally defined by Gavrilov [6] for meromorphic functions of the unit disk $\Delta$. The present version of $P$-sequence was introduced by Gauthier [7] in which he proved that the two notions are equivalent for meromorphic functions.

The following lemmas are key tools of this paper. 
Lemma 2.2 (Zalcman's Lemma [15]). Let $\mathcal{F}$ be a family of analytic functions in $\Delta$. Then $\mathcal{F}$ is not normal in $\Delta$ if and only if there exist (i) a number $r$ with $0<r<$ 1; (ii) points $z_{n}$ satisfying $\left|z_{n}\right|<r$; (iii) functions $f_{n} \in \mathcal{F}$; (iv) positive numbers $\rho_{n} \rightarrow 0$ as $n \rightarrow \infty$; such that

$$
f_{n}\left(z_{n}+\rho_{n} \xi\right) \rightarrow g(\xi) \text { as } n \rightarrow \infty
$$

uniformly on compact subsets of $\mathbb{C}$, where $g$ is a nonconstant entire function in $\mathbb{C}$. The function $g$ may be taken to satisfy the normalization $g^{\sharp}(z)<g^{\sharp}(0)=1 \quad(z \in \mathbb{C})$.

Here $g^{\sharp}(z)$ denotes the spherical derivative

$$
g^{\sharp}(z)=\frac{\left|g^{\prime}(z)\right|}{1+|g(z)|^{2}} .
$$

Lemma 2.3. Let $f$ be a normal function on a complex Banach manifold $X$ and suppose $k_{X}$ is a metric. There exists a constant $c>1$ such that

$$
\log (c \mu(f, x)) \leq \log (c \mu(f, y)) \exp ^{2 K_{X}(x, y)} \text { for all } x, y \in X .
$$

Here $\mu(f, x):=\max \{1,|f(x)|\}$.

This result was originally proved by Zaidenberg [17] in the case of complex manifolds. The proof of this result given in [9, page 39] extends immediately to the complex Banach manifold case.

\section{Normality and $P$-sequences}

In this section we obtain certain relations existing between notions of normal functions, and $P$-sequences.

Theorem 3.1. Let $X$ be a complex Banach manifold and suppose $k_{X}$ is a metric. The following statements are equivalent for $f \in \mathcal{O}(X)$ :

(a) $f$ is normal;

(b) there exists a constant $Q>0$ such that

$$
Q_{f}(x):=\sup _{v \in T_{x}(X) \backslash\{0\}} \frac{d s\left(f(x), f_{*}(x) v\right)}{k_{X}(x, v)}<Q \text { for all } x \in X ;
$$

(c) there exists a constant $L>0$ such that

$$
s(f(x), f(y)) \leq L \cdot K_{X}(x, y) \text { for all } x, y \in X
$$

(d) $f$ has no P-sequence. 
Proof. (a) $\Rightarrow$ (b): Assume that the family $\mathcal{F}=\{f \circ \varphi: \varphi \in \mathcal{O}(\Delta, X)\}$ is a normal family. By Marty's theorem [15, page 75], there exists a constant $L>0$ such that

$$
\frac{\left|(f \circ \varphi)^{\prime}(0)\right|}{1+|f \circ \varphi(0)|^{2}}<L \text { for all } \varphi \in \mathcal{O}(\Delta, X) \text {. }
$$

By the definition of $k_{X}$ there exists $\psi \in \mathcal{O}(\Delta, X)$ such that $\psi(0)=x, \psi_{*}(0) a=v$ for $a>0$ and $a / 2<k_{X}(x, v) \leq a$. Therefore, from (3.3),

$$
d s\left(f(x), f_{*}(x) v\right)<2 L \cdot k_{X}(x, v) \text { for all }(x, v) \in T(X) .
$$

Namely, $Q_{f} \leq 2 L$.

(b) $\Rightarrow$ (c): Let $x$ and $y$ be distinct points of $X$. It follows readily from (3.1) that

$$
d s\left(f(x), f_{*}(x) v\right)<Q \cdot k_{X}(x, v) .
$$

By integrating both side of the above inequality along the piecewise $C^{1}$ curves joining $x$ to $y$ in $X$ and by the definitions we have

$$
s(f(x), f(y)) \leq L \cdot \widetilde{K}_{X}(x, y) .
$$

Since $K_{X}$ is the integrated form of the infinitesimal metric $k_{X}$ (see [4, Corollary 3]) we have (3.2).

(c) $\Rightarrow$ (d): This follows immediately.

(d) $\Rightarrow$ (a): If $(d)$ holds, then the family $\mathcal{F}=\{f \circ \varphi: \varphi \in \mathcal{O}(\Delta, X)\}$ is equicontinuous at each point of $\Delta$, since otherwise there is a point $z_{0} \in \Delta$, some $\epsilon>0$, a sequence $\left\{z_{n}\right\}$ of points in $\Delta$ with $z_{n} \rightarrow z_{0}$, and a sequence $\left\{f \circ \varphi_{n}\right\} \subseteq \mathcal{F}$ satisfying

$$
s\left(f \circ \varphi_{n}\left(z_{n}\right), f \circ \varphi_{n}\left(z_{0}\right)\right) \geq \epsilon, n=1,2, \ldots .
$$

Since $K_{\Delta}=d_{\Delta}$, and $z_{n} \rightarrow z_{0}$ then an application in [3, Proposition 3.2] gives $K_{\Delta}\left(z_{n}, z_{0}\right) \rightarrow 0$, as $z_{n} \rightarrow z_{0}$. By the contracting property of the Kobayashi metric,

$$
K_{X}\left(\varphi\left(z_{n}\right), \varphi\left(z_{0}\right)\right) \leq K_{\Delta}\left(z_{n}, z_{0}\right) \rightarrow 0, \text { as } z_{n} \rightarrow z_{0} .
$$

From (3.4), (3.5) follows that sequence $\left\{\varphi_{n}\left(z_{0}\right)\right\}$ of points in $X$ is a $P$-sequence for $f$ which contradicts $(d)$.

Therefore, $\mathcal{F}=\{f \circ \varphi: \varphi \in \mathcal{O}(\Delta, X)\}$ is spherically equicontinuous family at each point of $\Delta$, and hence, by Montel's theorem [15, page 74], $\mathcal{F}$ is normal. This proves $(a)$.

Remark 3.2. Hahn [8] has also published a similar characterization of a normal function on a complex hyperbolic manifold $M$ of finite dimension. Unfortunately, Hahn's argument is incorrect, because the assumption that $\mathcal{F}=\{f \circ \varphi: \varphi \in$ $\mathcal{O}(\Delta, M)\}$ is not a equicontinuous family does not entail that "there exist an $\epsilon>0$ such that for all $n \in N$ there exist sequences $\left\{z_{n}\right\}$ and $\left\{w_{n}\right\}$ in $\Delta$ with $K_{\Delta}\left(z_{n}, w_{n}\right)<$ $1 / n$ but $s\left(f\left(\psi\left(z_{n}\right)\right), f\left(\psi\left(w_{n}\right)\right)\right) \geq \epsilon$ for some $\psi \in \mathcal{O}(\Delta, M)$ " (see [8, page 60]). 
Theorem 3.3. Let $X$ and $k_{X}$ be given as in Theorem 3.1 and let $f$ in $\mathcal{O}(X)$. If $Q_{f}\left(x_{m}\right) \rightarrow \infty$ as $m \rightarrow \infty$, then $\left\{x_{m}\right\}$ contains a subsequence which is a $P$ sequence of $f$.

Proof. Since $Q_{f}\left(x_{m}\right) \rightarrow \infty$ as $m \rightarrow \infty$, then by (3.1) there exist a sequences $\left\{v_{m}\right\}, v_{m} \in T_{x_{m}}(X)$, and $\left\{r_{m}\right\} \subset R, r_{m} \rightarrow \infty$ as $m \rightarrow \infty$, with

$$
d s\left(f\left(x_{m}\right), f_{*}\left(x_{m}\right) v_{m}\right)>r_{m} \cdot k_{X}\left(x_{m}, v_{m}\right) .
$$

By the definition of $k_{X}$ there exists $\psi_{m} \in \mathcal{O}(\Delta, X)$ such that $\psi_{m}(0)=x_{m}$, $\psi_{m *}(0) a_{m}=v_{m}$ for $a_{m}>0$ and $a_{m} / 2<k_{X}\left(x_{m}, v_{m}\right) \leq a_{m}$. Hence, inequality (3.6) gives,

$$
\frac{\left|\left(f \circ \psi_{m}\right)^{\prime}(0)\right|}{1+\left|f \circ \psi_{m}(0)\right|^{2}}>r_{m} \cdot \frac{k_{X}\left(x_{m}, v_{m}\right)}{a_{m}}>\frac{r_{m}}{2} \rightarrow \infty \text { as } m \rightarrow \infty .
$$

By Marty's theorem [15, page 75], the family $\left\{f \circ \psi_{m}\right\} \subseteq \mathcal{O}(\Delta)$ is not normal in any disc $\Delta_{\frac{1}{n}}=\left\{z \in \mathbb{C}:|z|<\frac{1}{n}\right\}$. Then by a local adaptation of the Zalcman Lemma $\left[15\right.$, page 152], there is a subsequence $\left\{f \circ \varphi_{n}\right\}$ of $\left\{f \circ \psi_{m}\right\}, z_{n} \rightarrow 0, \rho_{n} \rightarrow 0^{+}$ and a nonconstant entire function $g$ with $f \circ \varphi_{n}\left(z_{n}+\rho_{n} \xi\right) \rightarrow g(\xi)$ normally in $\mathbb{C}$. Passing to a subsequence if necessary, we can assume that $\left\{f \circ \varphi_{n}(0)\right\}$ converges to a some point $\beta \in \overline{\mathbb{C}}$. Set $g_{n}(\xi)=f \circ \varphi_{n}\left(z_{n}+\rho_{n} \xi\right)$. The sequence of functions $\left\{g_{n}\right\}$ converges locally uniformly to $g$. Let $\alpha$ be any complex number, $\alpha \neq \beta$, for which the equation $g(\xi)=\alpha$ has a solution $\xi_{0}$ which is not a multiple solution, that is, $g^{\sharp}\left(\xi_{0}\right) \neq 0$. By a theorem of Hurwitz [15, page 9], in each neighborhood of $\xi_{0}$ all but a finite number of the functions $\left\{g_{n}\right\}$ assume the value $\alpha$. Thus there exists a sequence of points $\left\{\xi_{n}\right\} \subseteq \mathbb{C}$ such that $\xi_{n} \rightarrow \xi_{0}$ and $g_{n}\left(\xi_{n}\right)=\alpha$ for $n$ sufficiently large. It follows $s\left(f \circ \varphi_{n}\left(z_{n}+\rho_{n} \xi_{n}\right), f \circ \varphi_{n}(0)\right) \geq s(\alpha, \beta) / 2>0$ for $n \geq N_{0}$. The Poincaré metric $d_{\Delta}$ is very closed to the Euclidian metric near 0 so $d_{\Delta}\left(0, z_{n}+\rho_{n} \xi_{n}\right) \rightarrow 0$ as $n \rightarrow \infty$. Hence $K_{X}\left(\varphi_{n}\left(z_{n}+\rho_{n} \xi_{n}\right), \varphi_{n}(0)\right) \leq$ $d_{\Delta}\left(z_{n}+\rho_{n} \xi_{n}, 0\right) \rightarrow 0$ as $n \rightarrow \infty$. Which proves that a subsequence $\left\{\varphi_{n}(0)\right\}_{n=N_{0}}^{\infty}$ of $\left\{x_{m}\right\}$ is a $P$-sequence of $f$. The proof is complete.

Remark 3.4. The converse to Theorem 3.3 is not true in general, as the following example [2, Example (4.3)] shows. Let $X=\Delta, f(z)=\exp \frac{i}{1-z} \in \mathcal{O}(\Delta)$, $z_{n}=\frac{n^{2}}{1+n^{2}}-\frac{i}{n+n^{3}}, w_{n}=\frac{n^{2}}{1+n^{2}}$. Here $Q_{f}\left(w_{n}\right) \rightarrow \infty$, and hence by Theorem 3.3 sequence $\left\{w_{n}\right\}$ contains a subsequence $\left\{w_{m}\right\}$ which is a $\mathrm{P}$-sequence of $f$. Since $K_{\Delta}\left(z_{m}, w_{m}\right) \rightarrow 0$ a subsequence $\left\{z_{m}\right\} \subseteq\left\{z_{n}\right\}$ is a P-sequence of $f$ too, while the sequence $Q_{f}\left(z_{m}\right) \rightarrow 0$.

\section{Criteria for non-normality}

The Zalcman Lemma [18] characterizing normal families of holomorphic functions on plane domains is the main tool used in proofs of theorems in this and next chapter. 
A relationship between non-normal holomorphic functions and the existence of a $P$-sequence is established in the following theorem.

Theorem 4.1. Let $X$ and $k_{X}$ be given as in Theorem 3.1. A function $f \in \mathcal{O}(X)$ is not a normal function on $X$ iff $f$ has a $P$-sequence.

Proof. If $f$ is not normal then the family $\mathcal{F}=\{f \circ \varphi: \varphi \in \mathcal{O}(\Delta, X)\}$ is not normal in $\Delta$. By Zalcman's Lemma, we can find $f \circ \varphi_{n} \in \mathcal{H}, z_{n} \subseteq \Delta_{r}, z_{n} \rightarrow z_{0}$, and $\rho_{n} \rightarrow 0^{+}$such that $f \circ \varphi_{n}\left(z_{n}+\rho_{n} \xi\right) \rightarrow g(\xi)$ uniformly on compacta, where $g$ is a nonconstant entire function on $\mathbb{C}$. Since $g$ is nonconstant in $\mathbb{C}$ there is $\zeta \in \mathbb{C}$ such that $g(0) \neq g(\zeta)$. One see at once that

$$
\lim _{n \rightarrow \infty} s\left(f \circ \varphi_{n}\left(z_{n}\right), f \circ \varphi_{n}\left(z_{n}+\rho_{n} \zeta\right)\right)=s(g(0), g(\zeta)) \geq \epsilon \text { for some } \epsilon>0
$$

and $K_{X}\left(\varphi_{n}\left(z_{n}\right), \varphi_{n}\left(z_{n}+\rho_{n} \zeta\right)\right) \leq d_{\Delta}\left(z_{n}, z_{n}+\rho_{n} \zeta\right) \rightarrow 0$ as $n \rightarrow \infty$. Hence a sequence $\left\{\varphi_{n}\left(z_{n}\right)\right\}$ of points in $X$ is a $P$-sequence of $f$.

Conversely, if $f$ have a $P$-sequence $\left\{x_{n}\right\}$, then there is a sequence $\left\{y_{n}\right\}$ of points in $X$ such that $\lim _{n \rightarrow \infty} K_{X}\left(x_{n}, y_{n}\right)=0$ but

$$
s\left(f\left(x_{n}\right), f\left(y_{n}\right)\right) \geq \epsilon \text { for some } \epsilon>0, n=1,2, \ldots .
$$

Suppose that $f$ is a normal function. By (3.2)

$$
s\left(f\left(x_{n}\right), f\left(y_{n}\right)\right) \leq L \cdot K_{X}\left(x_{n}, y_{n}\right) \text { for all } n \geq 1
$$

It follows $s\left(f\left(x_{n}\right), f\left(y_{n}\right)\right) \rightarrow 0$ as $n \rightarrow \infty$ which contradicts (4.1). Therefore $f$ is not a normal function. This proves the theorem.

A sufficient condition for the non-normality of a holomorphic function in $\Delta$ is given by Lappan [10, Lemma 3]. For holomorphic functions on a complex Banach manifold we have the following theorem.

Theorem 4.2. Let $X$ and $k_{X}$ be given as in Theorem 3.1. The following statements are equivalent for $f \in \mathcal{O}(X)$ :

(a) $f$ is not a normal function;

(b) There exist sequences $\left\{y_{m}\right\},\left\{x_{m}\right\}$ in $X$, and a constant $M>0$ such that $K_{X}\left(y_{m}, x_{m}\right)<M$ for all $m \geq 1, \lim _{m \rightarrow \infty} f\left(x_{m}\right)=\infty$, and $\lim _{m \rightarrow \infty} f\left(y_{m}\right)=$ $a \in \mathbb{C}$.

Proof. (a) $\Rightarrow$ (b): Since $h$ is not normal it follows that the family $\mathcal{F}=\{f \circ \varphi$ : $\varphi \in \mathcal{O}(\Delta, X)\}$ is not normal in $\Delta$. Apply the Zalcman Lemma to $\mathcal{F}$ with $r, z_{n}, \rho_{n}$, $f \circ \varphi_{n}$, and $g$ as given therein. Since $g$ is nonconstant entire function in $\mathbb{C}$ it follows that there exist a sequence $\left\{\xi_{m}\right\} \subset \mathbb{C}$ such that $\left|g\left(\xi_{m}\right)\right|>m$. In fact, if this were not the case, $g$ is bounded in $\mathbb{C}$. By Liouville's theorem $g \equiv$ constant, a contradictions 
with the assumption that $g$ is nonconstant entire function. For fixed $\xi_{m}$ chose $n_{m}$ large enough so that

$$
\begin{aligned}
& \text { (i) }\left|z_{n_{m}}+\rho_{n_{m}} \xi_{m}\right|<(1+r) / 2 \text {; } \\
& \text { (ii) }\left|f \circ \varphi_{n_{m}}\left(z_{n_{m}}+\rho_{n_{m}} \xi_{m}\right)\right|>m / 2 \text {. }
\end{aligned}
$$

Put $w_{n_{m}}=z_{n_{m}}+\rho_{n_{m}} \xi_{m}, x_{m}=\varphi_{n_{m}}\left(w_{n_{m}}\right)$, and $y_{m}=\varphi_{n_{m}}\left(z_{n_{m}}\right)$.

By the contracting property of the Kobayashi metric,

$$
K_{X}\left(y_{m}, x_{m}\right)=K_{X}\left(\varphi_{n_{m}}\left(w_{n_{m}}\right), \varphi_{n_{m}}\left(z_{n_{m}}\right)\right) \leq d_{\Delta}\left(w_{n_{m}}, z_{n_{m}}\right) .
$$

By the triangle inequality $d_{\Delta}\left(w_{n_{m}}, z_{n_{m}}\right) \leq d_{\Delta}\left(0, z_{n_{m}}\right)+d_{\Delta}\left(0, w_{n_{m}}\right)$. For any $z \in \Delta$ the Poincaré distance

$$
d_{\Delta}(0, z)=\frac{1}{2} \log \frac{1+|z|}{1-|z|} .
$$

Since $\left|z_{n_{m}}\right|<r$, and $\left|w_{n_{m}}\right|<(1+r) / 2$, we have

$$
d_{\Delta}\left(w_{n_{m}}, z_{n_{m}}\right) \leq \frac{1}{2}(\log ((1+r) /(1-r))+\log ((3+r) /(1-r))) .
$$

Putting the above together we get $K_{X}\left(y_{m}, x_{m}\right) \leq M$ where

$$
2 M=\log ((1+r) /(1-r))+\log ((3+r) /(1-r))<\infty .
$$

By (ii), we have that

$$
\lim _{m \rightarrow \infty} f\left(x_{m}\right)=\lim _{m \rightarrow \infty} f \circ \varphi_{n_{m}}\left(z_{n_{m}}+\rho_{n_{m}} \xi_{m}\right)=\infty .
$$

By (2.1), we have that

$$
\lim _{m \rightarrow \infty} f\left(y_{m}\right)=\lim _{m \rightarrow \infty} f \circ \varphi_{n_{m}}\left(z_{n_{m}}+\rho_{n_{m}} 0\right)=g(0) \in \mathbb{C} .
$$

(b) $\Rightarrow$ (a): Assume, to get a contradiction, that $f$ is normal. By Lemma 2.3,

$$
\log \left(c \mu\left(f, x_{m}\right)\right) \leq \log \left(c \mu\left(f, y_{m}\right)\right) \exp ^{2 K_{X}\left(x_{m}, y_{m}\right)} \text { for all } m \geq 1 .
$$

The left-hand side of the above inequality tends to infinity as $m \rightarrow \infty$ while the right-hand side tends to a number which is less than $\log (c \max \{1,|a|\}) \exp ^{2 r}$ and we have a contradiction. This contradiction proves the theorem. 


\section{Further results}

Theorem 5.1. Let $X$ and $k_{X}$ be given as in Theorem 3.1. If $f \in \mathcal{O}(X)$ is a normal function and if $h$ is a non-normal holomorphic function on $X$ such that each sequence $\left\{x_{m}\right\}$ of points in $X$ contains a subsequence $\left\{x_{m_{k}}\right\}$ on which at most one of $f$ or $h$ is unbounded, then $f+h$ is a non-normal function.

Proof. Since $h$ is not normal on $X$ it follows that the family $\mathcal{H}=\{h \circ \varphi: \varphi \in$ $\mathcal{O}(\Delta, X)\}$ is not normal in $\Delta$. By the Zalcman Lemma, we can find $h \circ \varphi_{n} \in \mathcal{H}$, $z_{n} \subseteq \Delta_{r}$, and $\rho_{n} \rightarrow 0^{+}$such that $h \circ \varphi_{n}\left(z_{n}+\rho_{n} \xi\right) \rightarrow g(\xi)$ uniformly on compacta, where $g$ is a nonconstant entire function on $\mathbb{C}$. But then

$$
\rho_{n} \frac{\left|h_{*}\left(\varphi_{n}\left(z_{n}+\rho_{n} \xi\right)\right) \varphi_{n}^{\prime}\left(z_{n}+\rho_{n} \xi\right)\right|}{1+\left|h\left(\varphi_{n}\left(z_{n}+\rho_{n} \xi\right)\right)\right|^{2}} \rightarrow g^{\sharp}(\xi) .
$$

We claim that there exist a sequence $\left\{\xi_{m}\right\} \subset \mathbb{C}$ such that $\left|g\left(\xi_{m}\right)\right|>m$ and $g^{\prime}\left(\xi_{m}\right) \neq 0$. Let us suppose not.

1. If $g^{\prime}(\xi) \neq 0$ and $g$ is bounded in $\mathbb{C}$ by Liouville's theorem $g \equiv$ constant, a contradictions with the assumption that $g$ is nonconstant entire function.

2. Let $\left\{a_{m}\right\}$ be the zeroes of $g^{\prime}(\xi)$. Suppose that $|g(\xi)|<L<\infty$ on $\mathbb{C} \backslash\{\xi \in$ $\left.\mathbb{C}: g^{\prime}(\xi)=0\right\}$. The zeros of $g^{\prime}(\xi)$ are isolated. Since nonconstant holomorphic function have no local maxima, we conclude that $\left|g\left(a_{m}\right)\right|<L$. Therefore $g$ is bounded in $\mathbb{C}$, hence constant by Liouville's theorem. But this contradicts with the assumption that $g$ is nonconstant entire function, and the assertion is proved.

For fixed $\xi_{m}$ chose $n_{m}$ large enough so that

$$
\begin{aligned}
& \text { (i) }\left|\lambda_{n_{m}}\right|<(1+r) / 2 \text {; } \\
& \text { (ii) } g^{\sharp}\left(\xi_{m}\right) / \rho_{n_{m}}>m / 2 \text {; } \\
& \text { (iii) } \rho_{n_{m}}\left|h_{*}\left(x_{m}\right) v_{m}\right| /\left(1+\left|h\left(x_{m}\right)\right|^{2}\right)>g^{\sharp}\left(\xi_{m}\right) / 2 \text {; } \\
& \text { (iv) }\left|h\left(x_{m}\right)\right|>m / 2 .
\end{aligned}
$$

Here $\lambda_{n_{m}}=z_{n_{m}}+\rho_{n_{m}} \xi_{m}, x_{m}=\varphi_{n_{m}}\left(\lambda_{n_{m}}\right)$, and $v_{m}=\varphi_{n_{m}}^{\prime}\left(\lambda_{n_{m}}\right)$. If (i), (ii), and (iii) hold, it is easy to see that

$$
\frac{\left|h_{*}\left(x_{m}\right) v_{m}\right|}{1+\left|h\left(x_{m}\right)\right|^{2}}>\frac{g^{\sharp}\left(\xi_{m}\right)}{2 \rho_{n_{m}}}>\frac{\left(4-(1+r)^{2}\right) m}{16} \cdot \frac{1}{1-\left|\lambda_{n_{m}}\right|^{2}} .
$$

By the distance decreasing property of the Kobayashi metric

$$
\frac{1}{1-\left|\lambda_{n_{m}}\right|^{2}}=k_{\Delta}\left(\lambda_{n_{m}}, 1\right) \geq k_{X}\left(\varphi_{n_{m}}\left(\lambda_{n_{m}}\right), \varphi_{n_{m}}^{\prime}\left(\lambda_{n_{m}}\right) \cdot 1\right)
$$


Hence

$$
\frac{\left|h_{*}\left(x_{m}\right) v_{m}\right|}{1+\left|h\left(x_{m}\right)\right|^{2}}>\frac{\left(4-(1+r)^{2}\right) m}{16} \cdot k_{X}\left(x_{m}, v_{m}\right) \text {. }
$$

Since $\left|h\left(x_{m}\right)\right| \rightarrow \infty$, as $m \rightarrow \infty$, by considering a subsequence if necessary, we may assume from the hypothesis of the theorem that $f$ is bounded on $\left\{x_{m}\right\}$, namely $\left|f\left(x_{m}\right)\right|<M<\infty$ for all $m \geq 1$, and hence, for $m$ sufficiently large, we have ${ }^{1}$

$$
\begin{gathered}
\frac{\left|(f+h)_{*}\left(x_{m}\right) v_{m}\right|}{1+\left|f\left(x_{m}\right)+h\left(x_{m}\right)\right|^{2}}>\frac{\left|h_{*}\left(x_{m}\right) v_{m}\right|-\left|f_{*}\left(x_{m}\right) v_{m}\right|}{2\left(1+\left|f\left(x_{m}\right)\right|^{2}\right)\left(1+\left|h\left(x_{m}\right)\right|^{2}\right)} \geq \\
\frac{1}{2}\left[\frac{1}{1+|M|^{2}} \cdot \frac{\left|h_{*}\left(x_{m}\right) v_{m}\right|}{1+\left|h\left(x_{m}\right)\right|^{2}}-\frac{\left|f_{*}\left(x_{m}\right) v_{m}\right|}{1+\left|f\left(x_{m}\right)\right|^{2}}\right] .
\end{gathered}
$$

By hypothesis, $f$ is a normal function on $X$ hence, by Theorem 3.1, there exists $Q>0$ such that $Q_{f}\left(x_{m}\right)<Q$ for all $m \geq 1$. Thus

$$
Q_{f+h}\left(x_{m}\right) \geq \frac{1}{2}\left[\frac{\left(4-(1+r)^{2}\right) m}{16\left(1+|M|^{2}\right)}-Q\right] \rightarrow \infty \text { as } m \rightarrow \infty
$$

By Theorem 3.1, $f+h$ is not a normal function on $X$.

Thus the proof is complete.

Lehto and Virtanen [12, page 53] remark that the sum of a normal function and a bounded function (which is necessary normal) is a normal function. The sum of two normal holomorphic functions, each omitting the values 0 and 1 , need not be normal (see [10, page 191]).

In the infinite dimensional case we have the following theorem.

Theorem 5.2. If under the conditions of Theorem 3.1, $f_{1}, \ldots, f_{l}$ are a finite number of normal holomorphic function on $X$ such that each sequence $\left\{x_{n}\right\}$ of points in $X$ contains a subsequence $\left\{x_{n_{m}}\right\}$ on which at most one of $f_{j}(1 \leq j \leq l)$ is unbounded, then $h:=\Sigma_{j=1}^{l} f_{j}$ is a normal function.

Proof. Suppose, on the contrary, that $h$ is not a normal function. By Theorem 4.2, we can find two sequences $\left\{x_{n}\right\}$ and $\left\{y_{n}\right\}$ of points in $X$, and a positive constant $M$ such that $K_{X}\left(x_{m}, y_{m}\right)<M$ for all $m \geq 1, \lim _{m \rightarrow \infty} h\left(x_{m}\right)=\infty$, and $\lim _{m \rightarrow \infty} h\left(y_{m}\right)=a \in \mathbb{C}$. Since $\lim _{m \rightarrow \infty} h\left(x_{m}\right)=\infty$ then $\left\{x_{m}\right\}$ contains a subsequence again denoted by $\left\{x_{m}\right\}$ such that at most one of $f_{j}$, say $f_{1}$, is unbounded

${ }^{1}$ If $a$ and $b$ in $\mathbb{C}$ then

$$
1+|a+b|^{2} \leq 1+(|a|+|b|)^{2}<2\left(1+|a|^{2}\right)\left(1+|b|^{2}\right) .
$$


on $\left\{x_{m}\right\}$. Since $\lim _{m \rightarrow \infty} h\left(y_{m}\right)=a \in \mathbb{C}$ then $\left\{y_{m}\right\}$ contains a subsequence $\left\{y_{m_{k}}\right\}$ such that either:

(i) at least two of $f_{j}(1 \leq j \leq l)$ is unbounded on $\left\{y_{m_{k}}\right\}$;

(ii) or $\lim _{k \rightarrow \infty} f_{j}\left(y_{m_{k}}\right)=\alpha_{j} \in \mathbb{C}(1 \leq j \leq l)$.

The case (i) is excluded by the assumption of the corollary.

Hence $\lim _{k \rightarrow \infty} f_{1}\left(x_{m_{k}}\right)=\infty, \lim _{k \rightarrow \infty} f_{1}\left(y_{m_{k}}\right)=\alpha_{1} \in \mathbb{C}$, and $K_{X}\left(x_{m_{k}}, y_{m_{k}}\right)<$ $M$ for all $k \geq 1$. By Theorem $4.2 f_{1}$ is not a normal function, a contradiction which proves the corollary.

Theorem 5.3. Under the assumption of Theorem 3.1 let $\left\{x_{n}\right\}$ and $\left\{y_{n}\right\}$ be two sequences of points in $X$, and let $M$ be a positive constant such that $K_{X}\left(x_{m}, y_{m}\right)<M$ for all $m \geq 1$. If $f \in \mathcal{O}(X)$ is a normal function which omits $l \in \overline{\mathbb{C}}$ in $X$ but $\lim _{m \rightarrow \infty} s\left(f\left(x_{m}\right), l\right)=0$ then $\lim _{m \rightarrow \infty} s\left(f\left(y_{m}\right), l\right)=0$.

Proof. Assume first that $l \in \mathbb{C}$. Since

$$
\frac{1+|f(x)-l|^{2}}{1+|f(x)|^{2}}<2\left(1+|l|^{2}\right)
$$

(see footnote 1) it follows from the hypothesis of the theorem and Theorem 3.1 that $g(x)=1 /(f(x)-l)$ is a normal holomorphic function on $X$. It is easy to see that $\lim _{m \rightarrow \infty} g\left(x_{m}\right)=\infty$. Therefore, we have $\lim _{m \rightarrow \infty} g\left(y_{m}\right)=\infty$ by Theorem 4.2. Hence $\lim _{m \rightarrow \infty} s\left(f\left(y_{m}\right), l\right)=0$ as desired.

If $l=\infty$ the corollary is an immediate consequence of Theorem 4.2. Thus the proof is complete.

\section{Boundary behavior of normal functions in a fixed boundary point}

In [1] F. Bagemihl and W. Seidel posed the following question: Given a sequence $\left\{z_{j}\right\} \subset \Delta$ converging to some $\varsigma \in \partial \Delta$ and a holomorphic mapping $f \in \mathcal{O}(\Delta, \overline{\mathbb{C}})$ such that $\lim _{j \rightarrow \infty} s\left(f\left(z_{j}\right), l\right)=0$ for some $l \in \overline{\mathbb{C}}$, under what conditions on $f$ and $\left\{z_{j}\right\}$ can $f$ have the limit l along some continuum in $\Delta$ which is asymptotic at $\varsigma$ ?

In this section, we give the infinite dimensional analogue of their result. First we introduce some definitions and prove two lemmas.

In the rest of the paper let $D$ be a bounded and convex domain in a complex Banach space $(V,\|\cdot\|)$, and $K_{D}$ be the Kobayashi metric for $D$.

A domain $D \subseteq V$ is called convex (in the real sense) if $(1-t) x+t y \subset D$ for all $x, y \in D$ and $0 \leq t \leq 1$.

Lemma 6.1. Let $D$ be a bounded and convex (in the real sense) domain in a complex Banach space $(V,\|\cdot\|)$ and let $\xi \in \partial D$. Then for all $a \in D$ the set $l_{\xi}(a)=\{y=\xi+t(a-\xi), 0<t<1\}$ is contained in D. 
Proof. We have $B(a, r):=a+B(0, r) \subset D$, for some $r>0$, where $B(0, r):=$ $\{y \in V:\|y\|<r\}$. Put $U=[t /(1-t)] B(0, r)+\xi, t \neq 1$. There is some $b \in D \cap U$. We have $b=[t /(1-t)] u+\xi$ where $u \in B(0, r)$. Since $a-u \in B(a, r) \subset D$ and $D$ is convex $y=(1-t) b+t(a-u)=\xi+t(a-\xi) \in D$. This proves that $l_{\xi}(a) \subset D$.

Definition 6.2. The approach region of aperture $\alpha$, with vertex $\xi \in \partial D$, and pole $a \in D$ is the set

$$
A_{\alpha}(\xi, a)=\left\{x \in D: K_{D}\left(x, l_{\xi}(a)\right)<\alpha\right\}
$$

where $K_{D}\left(x, l_{\xi}(a)\right)=\inf \left\{K_{D}(x, y), y \in l_{\xi}(a)\right\}$.

Lemma 6.3. Let $D$ be a bounded and convex (in the real sense) domain in a complex Banach space $V$ and let $\xi \in \partial D$. If $b \in D, b \neq a$, then there exists a positive constant $C$ such that

$$
A_{\alpha}(\xi, a) \subset A_{\alpha+C}(\xi, b) \subset A_{\alpha+2 C}(\xi, a) .
$$

Proof. To prove the lemma it suffices to show that if $z \in A_{\alpha}(\xi, a)$ then $z \in$ $A_{\alpha+C}(\xi, b)$. So let $z \in A_{\alpha}(\xi, a)$. From the definition of $A_{\alpha}(\xi, a)$ follows that for every $\varepsilon>0$ there is a point $a_{1}$ in $l_{\xi}(a)$ such that $K_{D}\left(z, a_{1}\right)<\alpha+\varepsilon$. Since $a_{1} \in l_{\xi}(a)$ there exists a $\tau_{0} \in(0,1]$ such that $a_{1}=\xi+\tau_{0}(a-\xi)$. Set $b_{1}=\xi+\tau_{0}(b-\xi) \in l_{\xi}(b)$.

Let $\varphi$ be a mapping in $\mathcal{O}(\Delta, D)$ whose range contains both $a$ and $b .^{2}$ Suppose that $\varphi(0)=a$ and $\varphi(\zeta)=b$ for a suitable $\zeta \in \Delta$. Define $\psi: \Delta \rightarrow V$ by

$$
\psi(z)=\xi+\tau_{0}(\varphi(z)-\xi) .
$$

By Lemma $6.1 \psi$ maps $\Delta$ into $D$; hence

$$
K_{D}\left(a_{1}, b_{1}\right)=K_{D}(\psi(0), \psi(\zeta)) \leq d_{\Delta}(0, \zeta) .
$$

By the triangle inequality of the Kobayashi distance

$$
K_{D}\left(z, b_{1}\right) \leq K_{D}\left(z, a_{1}\right)+K_{D}\left(a_{1}, b_{1}\right) .
$$

It is clear, that

$$
K_{D}\left(z, l_{\xi}(b)\right) \leq K_{D}\left(z, b_{1}\right) \leq \alpha+\varepsilon+d_{\Delta}(0, \zeta)
$$

${ }^{2}$ If $D \subset \mathbb{C}^{n}$ then there exists a continuous path $\phi:[0,1] \rightarrow D$ such that $\phi(0)=a$ and $\phi(1)=b$. An application of the vector-valued Stone-Weierstrass theorem and an end-point adjustment shows that we can take $\phi$ to be a polynomial. By compactness there exists a convex open neighborhood $O$ of $[0,1]$ in $\mathbb{C}$ such that $\widehat{\phi}(O) \subset D$, where $\widetilde{\phi}$ is the polynomial on $\mathbb{C}$ obtained by replacing the real variable $x$ by the complex variable $z$. An application of the Riemann mapping theorem yields the existence of a mapping $\varphi \in \mathcal{O}(\Delta, D)$ whose range contains both $a$ and $b$.

Since $D$ is a domain in a Banach space $V$ then any pair of points $a$ and $b$ in $D$ can be joined by a polygonal curve $\Gamma$. If $Y$ is the finite dimensional subspace of $V$ spanned by the points of $\Gamma$ then the connected component of $\Gamma \cap D$, containing $a$, is a domain in a finite dimensional space which contains both $a$ and $b$. The finite dimensional result can now be applied to show the existence of a mapping $\phi \in \mathcal{O}(\Delta, D)$ whose range contains both $a$ and $b$ (see [3, page 49]). 
Since $\varepsilon$ was arbitrary this gives that

$$
K_{D}\left(z, l_{\xi}(b)\right) \leq \alpha+d_{\Delta}(0, \zeta) .
$$

It follows

$$
z \in A_{\alpha+C}(\xi, b)
$$

where $C=d_{\Delta}(0, \zeta)<\infty$.

Remark 6.4. If $D$ is the unit ball in $\mathbb{C}^{n}$ then the approach regions $A_{\alpha}(\xi, a)$ are comparable to the admissible approach regions of Koranyi.

Definition 6.5. A function $f \in \mathcal{O}(D)$ has an admissible limit $l \in \overline{\mathbb{C}}$ at $\xi \in \partial D$, if for every $\alpha>0$ and every sequence of points $\left\{x_{n}\right\} \subset A_{\alpha}(\xi, a)$ which tends to $\xi$

$$
\lim _{n \rightarrow \infty} s\left(f\left(x_{n}\right), l\right)=0 .
$$

Remark 6.6. From Lemma 6.3 follows that the definition of admissible limit is independent of the choice of the pole $a \in D$.

Theorem 6.7. Let $D$ be a bounded and convex (in the real sense) domain in a complex Banach space $(V,\|\cdot\|)$. Let $\left\{x_{n}\right\}$ be a sequence of points in $l_{\xi}(a)$ which tends to $\xi \in \partial D$, such that there exists a constant $\varepsilon>0$ such that $K_{D}\left(x_{n}, x_{n+1}\right)<$ $\varepsilon$ for all $n \geq 1$. Suppose that the function $f \in \mathcal{O}(D)$ is normal on $D$, omits $l \in \overline{\mathbb{C}}$ in $D$ but

$$
\lim _{n \rightarrow \infty} s\left(f\left(x_{n}\right), l\right)=0 .
$$

Then $f$ has an admissible limit $l$ at $\xi$.

Proof. First of all, we show that for an arbitrary sequence of points $\left\{q_{n}\right\}$ in $l_{\xi}(a)$ which tends to $\xi$ as $n \rightarrow \infty$ we have $\lim _{n \rightarrow \infty} s\left(f\left(q_{n}\right), l\right)=0$. Indeed, for every $q_{n}$ there exists an integer $j_{n}$ and a constant $t_{n}, t_{n} \in[0,1]$, such that $q_{n}=x_{j_{n}}+$ $t_{n}\left(x_{j_{n}+1}-x_{j_{n}}\right)$. For $t \in[0,1]$ define $g_{t} \in \mathcal{O}((D \times D) \times(D \times D), D \times D)$ by $g_{t}((x, y),(w, z))=(t x+(1-t) y, t w+(1-t) z)$. Then by [5, Proposition IV.1.2, page 83] for all $x, y, w, z \in D$ we have

$$
K_{D \times D}((x, y),(w, z)) \geq K_{D}(t x+(1-t) y, t w+(1-t) z) .
$$

By [5, Proposition V.4.2, page 136]

$$
K_{D \times D}((x, y),(w, z))=\max \left\{K_{D}(x, y), K_{D}(w, z)\right\} .
$$

Combining this with (6.1), we see that

$$
K_{D}(t x+(1-t) y, t w+(1-t) z) \leq \max \left[K_{D}(x, w), K_{D}(y, z)\right]
$$

for all $0 \leq t \leq 1$ and every choice of $x, y, w, z$ from $D$. Then we have

$$
K_{D}\left(x_{j_{n}}, q_{n}\right)=K_{D}\left(x_{j_{n}}, x_{j_{n}}+t_{n}\left(x_{j_{n}+1}-x_{j_{n}}\right) \leq K_{D}\left(x_{j_{n}}, x_{j_{n}+1}\right)<\varepsilon .\right.
$$


As $\lim _{n \rightarrow \infty} s\left(f\left(x_{j_{n}}\right), l\right)=0$ and $K_{D}\left(x_{j_{n}}, q_{n}\right)<\varepsilon$ for all $n \geq 1$, we get

$$
\lim _{n \rightarrow \infty} s\left(f\left(q_{n}\right), l\right)=0
$$

by Corollary 5.3.

Let $\left\{y_{n}\right\}$ be any sequence in $A_{\alpha}(\xi, a)$ which converges to the point $\xi \in \partial D$. For every point $y_{n}$ there exists a point $b_{n} \in l_{\xi}(a)$ such that $2 \alpha>K_{D}\left(y_{n}, b_{n}\right)$ for all $n \geq 1$. Every $b_{n}$ can be write in the form $b_{n}=\xi+\tau_{n}(a-\xi)$ where $\tau_{n} \in[0,1]$. Hence, $\left\{b_{n}\right\}$ has a convergent subsequence $\left\{b_{n_{j}}\right\}$. Let $b=\xi+\tau_{0}(a-\xi)$ be a limit point of $\left\{b_{n_{j}}\right\}$.

Assuming $\tau_{0} \neq 0$ we shall derive a contradiction. Choose a positive number $r$ such that $B_{r}(b)=\{y \in V:\|y-b\|<r\} \subset D$. Since sequence $\left\{b_{n_{j}}\right\}$ tends to $b$ as $j \rightarrow \infty$ there exits an integer $j_{0}$ such that $\left\|b_{n_{j}}-b\right\|<r / 2$ for all $j \geq j_{0}$. By the inequality (4.3) in [3, page 52$]$

$$
K_{D}\left(b_{n_{j}}, b\right)<\tanh ^{-1}\left(\frac{1}{2}\right) \text { for all } j \geq j_{0} .
$$

Then for $j \geq j_{0}$ we have

$$
K_{D}\left(y_{n_{j}}, b\right)<K_{D}\left(y_{n_{j}}, b_{n_{j}}\right)+K_{D}\left(b_{n_{j}}, b\right)<2 \alpha+\tanh ^{-1}\left(\frac{1}{2}\right) .
$$

This contradicts our assumption that sequence $\left\{y_{n}\right\}$ tends to $\xi \in \partial D$, since every ball in $\left(D, K_{D}\right)$ is bounded away from the boundary (see [3, page 88]). Hence $\tau_{0}=0$ and sequence $\left\{b_{n}\right\} \subset l_{\xi}(a)$ tends to $\xi$ as $n \rightarrow \infty$. As proved above $\lim _{n \rightarrow \infty} s\left(f\left(b_{n}\right), l\right)=0$ and since $K_{D}\left(y_{n}, b_{n}\right)<\alpha$ for all $n \geq 1$

$$
\lim _{n \rightarrow \infty} s\left(f\left(y_{n}\right), l\right)=0
$$

by Corollary 5.3. Hence $f$ has the admissible limit $l$ at $\xi$.

\section{References}

[1] F. BAgeminl and W. SEIDEL, Sequential and continuous limits of meromorphic functions, Ann. Acad. Sci. Fenn. Math. 280 (1960).

[2] D. M. CAMPBEll and G. WiCKES, Characterizations of normal meromorphic functions, In: "Complex Analysis", Laine, J. et al. (eds.), Joensuu 1978, Lect. Notes Math., Vol. 747, Springer, Berlin-Heidelberg-New York, 1979, 55-72.

[3] S. DineEn, "The Schwarz Lemma", Oxford Mathematical Monographs, Oxford, 1989.

[4] C. J. Earle, L. A. Harris, J. H. Hubbard and S. Mitra, Schwarz's lemma and the Kobayashi and Carathéodory metrics on complex Banach manifolds, In: "Kleinian Groups and Hyperbolic 3-Manifolds", Cambridge Univ. Press, Cambridge, Lond. Math. Soc. Lec. Notes, Vol. 299, 2003, 363-384. http://www.ms.uky.edu/ larry/paper.dir/minsky.ps.

[5] T. Franzoni and E. Vesentini, "Holomorphic Maps and Invariant Distances", NorthHolland Mathematical Studies 40, North-Holland Publishing, Amsterdam, 1980. 
[6] V. I. GaVRILov, Boundary properties of functions meromorphic in the unit disc, Dokl. Akad. Nauk SSSR 151 (1963), 19-22 (in Russian).

[7] P. GAUTHIER, A criterion of normalcy, Nagoya Math. J. 32 (1968), 272-282.

[8] K. T. HAHN, Non-tangential limit theorems for normal mappings, Pacific J. Math. 135 (1988), 57-64.

[9] M. H. KWACK, "Families of Normal Maps in Several Variables and Classical Theorems in Complex Analysis", Lecture Notes Series, Vol. 33, Res. Inst. Math., Global Analysis Res. Center, Seoul, Korea, 1996.

[10] P. LAPPAN, Non-normal sums and prodacts of unbounded normal functions, Michigan Math. J. 8 (1961), 187-192.

[11] P. LAPPAN, Normal families and normal functions: results and techniques, In: "Function Spaces and Complex Analysis", Joensuu 1997, Univ. Joensuu, Department of Mathematics Rep. Ser. 2 (1997), 63-78.

[12] O. LEHTO and K. I. VIRTANEN, Boundary behaviour and normal meromorphic functions, Acta Math. 97 (1957), 47-65.

[13] A. J. LohwATER, The boundary behavior of analytic functions, In: "Current Problems in Mathematics, Fundamental Directions", Vol. 10, Itogi Nauki i Tekhniki, Akad. Nauk SSSR, Vsesoyuz. Inst. Nauchn. i Tekhn. Inform., Moscow, 1973, 99-259 (in Russian).

[14] K. Noshiro, Contributions to the theory of meromorphic functions in the unit circle, J. Fac. Sci. Hokkaido Imp. Univ., Ser. I (1938), 149-159.

[15] J. L. SHCHIFF, "Normal Families", Springer, New York, 1993.

[16] K. Yosida, On a class of meromorphic functions Proc. Phys.-Math. Soc. Japan, ser. 1, 3 (1934), 227-235.

[17] M. G. ZAIDENBERG, Schottky-Landau growth estimates for s-normal families of holomorphic mappings, Math. Ann. 293 (1992), 123-141.

[18] L. Zalcman, Normal families: new perspectives, Bull. Amer. Math. Soc. 35 (1998), 215230.

Institute of Mathematics

and Computer Science of the Academy

of Sciences of Moldova

5 Academy Street

Kishinev, MD-2028, Republic of Moldova peter.dovbush@gmail.com 